\title{
Metodologías ágiles en el grado de Tecnologías interactivas de la Escuela Politècnica Superior de Gandia
}

\author{
José Luis Giménez ${ }^{a}$, Daniel Palacio ${ }^{b}$, Francisco de Zuluetac, Rebeca Diez ${ }^{\mathrm{d}}$, Rafael \\ Seiz ${ }^{\text {, }}$, Jesus Garcia Laborda ${ }^{g}$ \\ jogilo@upvnet.upv.es, bdapasa@har.upv.es, 다razudo@har.upv.es ${ }^{\mathrm{d}}$ rdiez@har.upv.es, \\ emarch@dib.upv.es, rsseiz@upvnet.upv.es \\ Universitat Politècnica de València \\ g.jesus.garcialaborda@uah.es \\ Universidad de Alcalá
}

\begin{abstract}
In view of the continuous advance of newer technologies, higher education in engineering should transmit not only the methodological and developmental aspects of a conventional project, but the need for adaptation and continuous learning in the labour market. As a result, the new degree on Interactive Technologies B.E. at the Higher Polytechnic School of Gandia (EPSG) has been designed taking as reference the work oriented towards project-based learning (PBL), CDIO Initiative (Conceive Design Implement Operate), and a learning-teaching methodology based on Scrum. It is with these implementations that we hope to approach the working procedures that highperformance companies carry out: the so-called agile methodologies. As per the case, we can observe how the aforementioned methodologies have been integrated to higher education and, in particular, to the workshop "Project: Web Design and Development", which belongs to the second semester of the first bachellor's year. In this article we describe how the learning-teaching methodology (Scrum) has been introduced to the academic planning, the subject structure and methodology, as well as to the student competences, their avaluation and rubrics.
\end{abstract}

Keywords: agile methodologies, interactive technologies, problem-based learning, CDIO Initiative, Scrum

\footnotetext{
Resumen

Ante el avance de las nuevas tecnologías, la enseñanza universitaria en el ámbito de las ingenierías debe transmitir al alumno, además de los aspectos metodológicos y de desarrollo de un proyecto convencional, la necesidad de adaptación y aprendizaje continuado en el mundo laboral. En consecuencia, el nuevo grado de Tecnologías interactivas de la Escuela Politècnica Superior de Gandia (EPSG) se ha diseñado tomando como referencia el trabajo orientado a proyectos (PBL), la filosofía CDIO (concebir, diseñar, implementar y operar) y una metodología de aprendizaje-enseñanza basada en Scrum. Con estas implementaciones se pretende una aproximación a los procedimientos de trabajo que se realizan en empresas tecnológicas de alto
} 
Metodologías ágiles en el grado de Tecnologías interactivas de la Escuela Politècnica Superior de Gandia

rendimiento: las denominadas metodologías ágiles. En el caso que nos ocupa, podemos observar cómo dichas metodologías se han incorporado a la enseñanza universitaria $y$, en concreto, a la asignatura-taller "Proyecto: diseño y programación web", perteneciente al segundo semestre de primer año del grado. En el presente artículo se describen las adaptaciones del Plan de Ordenación Docente, de la estructura y metodología de la asignatura, así como de las competencias del alumno, de su evaluación y rúbricas, a la nueva metodología activa (Scrum) de enseñanza-aprendizaje.

Palabras clave: Metodologías ágiles, tecnologías interactivas, PBL, CDIO, SCRUM.

\section{Introducción}

En los últimos años prestigiosas universidades de todo el mundo (MIT, Aalborg, etc.) han implementado títulos utilizando metodologías como el aprendizaje basado en proyectos (PBL) dónde la formación en competencias transversales se integra de una forma racional con la formación en competencias específicas a través del desarrollo de proyectos de aplicación práctica en el mundo real, (Markham et. Al. 2003), (Baviera et Al, 2009).

Tomando esto como modelo se ha implementado en el Grado en Tecnologías Interactivas de la Escuela Politècnica Superior de Gandia de la UPV. Con ello se pretende dotar a sus estudiantes de las competencias específicas aplicando la filosofía CDIO: concibiendo, diseñando, implementando y operando productos y servicios tecnológicos a través de proyectos semestrales tematizados en las tecnologías que han emergido en los últimos tiempos y en otras nuevas que irán apareciendo, (CDIO, 2018). Con la implementación de la metodología de trabajo orientada a proyectos (PBL) (Magal-Royo et $\mathrm{Al}, 2006$ ), se pretende la formación integral de los estudiantes en las competencias transversales citadas y demandadas por las empresas y la evaluación de las mismas de forma objetiva a lo largo de los cuatro cursos del grado de Tecnologías Interactivas, (Philips, 1994), (Fernández et Al. 2003).

Para el correcto aprendizaje de métodos de trabajo, que se integren en el mundo empresarial, se ha optado por aplicar las metodologías ágiles. En concreto la metodología SCRUM, basada en el manifiesto Agile, (The Agile Manifiesto, 2018), (SCRUM, 2018). Este es un proceso en el que se aplica una metodología donde se trabaja colaborativamente, en equipo, para obtener un producto mínimo viable en cortos periodos de tiempo. Estas prácticas se basan en procesos iterativos, flexibles y altamente dinámicos que permiten obtener resultados ajustados a las necesidades del cliente. Procesos que se dan en equipos de trabajo altamente productivos de la empresa privada, (Tore y Torgeir,2008), (Salas et Al. 2009).

(c) EY-NC-ND 2018, Universitat Politècnica de València

Congreso IN-RED (2018) 


\section{Objetivos.}

Este documento pretende mostrar como se pueden implementar las metodologías ágiles empleadas en el mundo profesional en la enseñanza universitaria. En concreto en la asignatura de proyecto diseño y programación web del grado de tecnologías interactivas, que se imparte en la Escuela Politécnica Superior de Gandia. Este grado es de reciente creación, actualmente se imparte primer curso. Es el primero en España en realizar una formación por proyectos.

\section{Desarrollo de la innovación.}

En esta asignatura los alumnos desarrollan un proyecto que consiste en crear un site, según la metodología CDIO. Esta metodología se basa en una premisa comúnmente aceptada de que los graduados de ingeniería deberían ser capaces de: Concebir - Diseñar - Implementar - Operar sistemas complejos de ingeniería con valor agregado en un ambiente moderno y basado en el trabajo en equipos para crear sistemas y productos, (Rico y Sayani,2009).

La iniciativa CDIO ofrece entonces un modelo educativo que enfatiza los fundamentos de la ingeniería, en el contexto de la Concepción - Diseño - Implementación - Operación de procesos. Esta iniciativa es rica en proyectos estudiantiles con una gran carga practica. Implementa experiencias de aprendizaje activo grupal tanto en clases como en talleres de aprendizaje y laboratorios, además de valoración y procesos de evaluación, esto favorece un alto grado de motivación en el alumnado, (Ampuero et Al, 2011).

Los objetivos de la iniciativa CDIO son:

Educar a los estudiantes para el dominio profundo y aplicado de los fundamentos técnicos.

- Educar a los ingenieros para liderar en la creación y operación de nuevos productos y sistemas.

- Educar futuros investigadores para comprender la importancia y valor estratégico de su trabajo.

La iniciativa CDIO fue diseñada específicamente como un modelo que puede ser adaptado y adoptado por cualquier escuela universitaria de ingeniería (Manamendra, et Al., 2013). Dado que CDIO es un modelo de arquitectura abierta, está disponible para ser adaptado a las necesidades específicas de todos los programas de ingeniería universitarios. Las universidades participantes en esta iniciativa, desarrollan regularmente material y metodologías para compartir con otros, (Sherwood, 2004).

El grado de Tecnologías Interactivas esta basado desde sus inicios en esta filosofía CDIO.

En el primer semestre se imparten asignaturas básicas donde se le da al alumno un conocimiento teórico con asignaturas como: Álgebra matricial y geometría, Electrónica básica, Fundamentos físicos y Programación donde el alumno comprende y utiliza los principios de diseño arquitectónico y algorítmico para desarrollar aplicaciones, mediante el uso de un lenguaje de programación. Otra asignatura es Desarrollo de un proyecto electrónico utilizando metodología CDIO esta asignatura tiene una carga de 6 créditos 
Metodologías ágiles en el grado de Tecnologías interactivas de la Escuela Politècnica Superior de Gandia

ECTS. En este proyecto los alumnos han desarrollado unos sensores que permiten monitorizar una serie de parámetros en un campo. A partir de este proyecto nuestra asignatura de segundo semestre desarrolla un site donde muestra todos los datos que recogen los sensores en tiempo real. Permitiendo monitorizar el campo vía web.

La asignatura que nos ocupa (13949) “Proyecto diseño y programación web” es de carácter obligatorio. Pertenece al Módulo 4 Proyectos de las tecnologías interactivas, materia 13Proyecto Diseño y Programación Web. Se imparte en el primer curso segundo semestre. Tiene asignados 12 créditos, 6 de teoría y 6 de prácticas. Junto a ella también se imparten otras asignaturas como Diseño de interfaces y experiencias de usuario con 6 créditos, Programación 2 (6 créditos), Redes y servicios telemáticos. Estas asignaturas apoyan al proyecto de diseño y programación web.

Nuestra asignatura se desarrolla según los patrones de CDIO. Esto comprende analizar, planificar, organizar, desarrollar y diseñar el site, observando la usabilidad y la experiencia de usuario. A la hora de realizar el proyecto deben tener en cuenta el diseño de la aplicación front-end (hace referencia a la visualización del usuario por un lado), y del back-end (administrador del sitio con sus respectivos sistemas por el otro).

Al final se procederá a publicar el sitio y promocionarlo en la red. Con esto se observará el proceso completo de creación de un sitio web. Y podrá integrarse en un equipo de trabajo profesional.

\section{Metodología y métodos}

La educación en ingeniería y las demandas del mundo real sobre los ingenieros se han separado en los últimos años. Al darse cuenta de que esta creciente brecha debe ser zanjada, las principales escuelas de ingeniería de Estados Unidos, Europa, Canadá, Reino Unido, África, Asia y Nueva Zelanda formaron la Iniciativa CDIO: Una colaboración a nivel mundial para concebir y desarrollar una nueva visión de la enseñanza de la ingeniería, (Rousseau, 2012), (Rousseau y McCarthy, 2007).

Como indicamos anteriormente la iniciativa CDIO ofrece entonces un modelo educativo que enfatiza los fundamentos de la ingeniería, en el contexto de la Concepción - Diseño Implementación - Operación de procesos.

El proceso de aprendizaje-enseñanza estaría basado en la metodología SCRUM. Este es un proceso en el que se aplican de manera regular un conjunto de buenas prácticas para trabajar colaborativamente, en equipo, y obtener el mejor resultado posible de un proyecto. Estas prácticas se apoyan unas a otras y su selección tiene origen en un estudio de la manera de trabajar de equipos altamente productivos.

En SCRUM se realizan entregas parciales y regulares del producto final, priorizadas por el beneficio que aportan al receptor del proyecto. Por ello, Scrum está especialmente indicado para proyectos en entornos complejos, donde se necesita obtener resultados de forma rápida, donde los requisitos son cambiantes o poco definidos, donde la innovación, la competitividad, la flexibilidad y la productividad son fundamentales, (Hammersley, 2004).

(cc) EY-NC-ND 2018, Universitat Politècnica de València

Congreso IN-RED (2018) 


\section{Estructuración}

Los contenidos se dividen en seis apartados (ver tabla 1), fundamentales para la realización de un proyecto web. El primero de todos se centra en explicar los fundamentos del proyecto, su diseño, desarrollo y su gestión, para dejar definidos los roles y sus características. El segundo bloque introduce a los alumnos en el desarrollo front-end y back-end, estableciendo las pautas para un buen desarrollo. En el tercer bloque entramos de lleno en el desarrollo front-end a partir del lenguaje HTML, css y JavaScript. En el cuarto bloque pasamos a las tecnologías Back-end, lenguajes de servidor y bases de datos. Para en un quinto bloque publicar en la red nuestro proyecto. Pero no debemos olvidar el marketing, la promoción de nuestro site para que llegue al mercado en las mejores condiciones, que es lo que se trabajará en el sexto y último bloque.

Tabla 1. Estructuración de las unidades docentes de la asignatura.

1. Fundamentos diseño, desarrollo y gestión de un proyecto web

Diseño de un Product Backlog. Personas. Historias de usuario. Mapa de historias

Diseño incremental. Refactorización

Roles y tareas en un proyecto web

2. Introducción a Fundamentos del front-end y back-end (cliente servidor)

Arquitectura cliente-servidor

Direcciones y puertos IP. DNS

Fundamentos del protocolo HTTP

Instalación y gestión básica de un servidor web

Páginas estáticas y páginas dinámicas

3. Tecnologías implicadas en el desarrollo front-end

HTML estructura semántica

CSS: el diseño del front-end

JavaScript: el comportamiento del front-end

4. Tecnologías implicadas en el desarrollo back-end

Lenguajes de servidor y bases de datos

Envío de información al servidor. Métodos GET y POST

5. Tecnologías implicadas en la publicación

Gestión de usuario

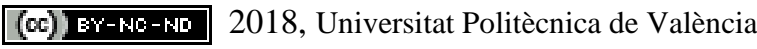

Congreso In-Red (2018) 
Metodologías ágiles en el grado de Tecnologías interactivas de la Escuela Politècnica Superior de Gandia

\section{Marketing, análisis y promoción web}

$$
\text { Promoción y reputación online }
$$

$$
\text { Plan de marketing online }
$$

Técnicas para fidelizar y uso de las redes sociales

Fuente: EICE, ABP-Diseño y Comunicación, 2018

A continuación, se detalla la distribución de las horas respecto a las unidades didácticas (Ver tabla 2).

\begin{tabular}{|c|c|c|c|c|c|c|}
\hline $\begin{array}{l}\text { Unidad } \\
\text { Didáctica }\end{array}$ & $\begin{array}{c}\text { Teoría de } \\
\text { Aula }\end{array}$ & $\begin{array}{l}\text { Práctica de } \\
\text { Laboratorio }\end{array}$ & $\begin{array}{c}\text { Actividades } \\
\text { de } \\
\text { Evaluación }\end{array}$ & $\begin{array}{l}\text { Trabajo } \\
\text { Presencial }\end{array}$ & $\begin{array}{l}\text { Trabajo No } \\
\text { Presencial }\end{array}$ & $\begin{array}{l}\text { Total } \\
\text { horas }\end{array}$ \\
\hline 1 & 15 & 12 & 6 & 33 & 30 & 63 \\
\hline 2 & 10 & 12 & 2 & 24 & 25 & 49 \\
\hline 3 & 20 & 16 & 2 & 38 & 60 & 98 \\
\hline 4 & 5 & 10 & 3 & 18 & 40 & 58 \\
\hline 5 & 5 & 5 & 1 & 11 & 25 & 36 \\
\hline 6 & 5 & 5 & 1 & 11 & 25 & 36 \\
\hline Total horas & 60 & 60 & 15 & 135 & 205 & 173 \\
\hline
\end{tabular}

Tabla 2. Distribución de las horas según las unidades docentes.

Fuente: EICE, ABP-Diseño y Comunicación, 2018

La asignatura está compuesta de distintos talleres que imparten distintos departamentos, acordes con la temática específica. (Ver tabla 3)

Tabla 3. Distribución de los créditos según los departamentos que intervienen

\begin{tabular}{|c|c|c|c|}
\hline MODULOS & TEORÍA & PRACTICA & TOTAL \\
\hline Seguimiento Proyecto (DIG) & 1,5 & 0,5 & 2 \\
\hline Análisis y diseño (DIG) & 2 & 1 & 3 \\
\hline Front-end (DCADHA) & 0,5 & 2,5 & 3 \\
\hline Back-end (DSIC) & 1,5 & 1 & 2,5 \\
\hline Promoción y seguimiento (DCADHA) & 0,5 & 1 & 1,5 \\
\hline Total proyecto & 6 & 6 & 12 \\
\hline
\end{tabular}

Fuente: EICE, ABP-Diseño y Comunicación, 2018 


\section{Evaluación}

Los estudiantes desarrollan un producto nuevo y único mediante la realización del proyecto. Este recogerá lo aprendido durante el semestre. Será la última prueba evaluativa y se entregará en la última semana del curso, (Guo et Al., 2016).

Para la realización del proyecto se crearán grupos de alumnos, que trabajarán en la realización del mismo a lo largo del semestre. El método de realización seguirá la metodología scrum. Esta metodología se basará en el CDIO (Concebir, Diseñar, Implementar y Operar).

Durante el primer mes se realizará el trabajo académico este tendrá un valor del 10\% de la nota global. A partir de ese mes se establecerán sprints de dos semanas, realizándose un total de 6 sprints. Siendo el último la presentación en exposición pública del proyecto. Los cinco primeros sprints (Examen oral), tendrán un valor cada uno del 8\% siendo el total de $40 \%$. Otro $10 \%$ será aportado por el método de observación durante el trabajo en los diferentes talleres.

El último sprint tendrá un valor del 40\%, donde se valorará la resolución del proyecto, la memoria aportada y su exposición. Dividiéndose la nota final obtenida entre todos ellos según el trabajo realizado. La evaluación final será la nota ponderada de todos los actos evaluativos. Ver tabla 4.

El proyecto será evaluado por un comité de profesores que ha ido impartiendo los contenidos necesarios para realizar el producto. Esta evaluación se realizará en una presentación pública conjunta, ante los demás miembros de la clase. La exposición se realizará durante la primera semana de la convocatoria oficial de exámenes, al final del cuatrimestre. La segunda semana se destinará a recuperaciones en el caso que el comité considere que es necesario recuperar alguna parte del proyecto del último sprint.

Los alumnos a los que se les conceda la dispensa de asistencia a actividades presenciales deberán integrarse en un equipo de trabajo desde el comienzo del cuatrimestre, utilizando para ello herramientas de trabajo colaborativo y asistiendo a las reuniones de manera virtual (por Skype, Polireunión etc.).

La asistencia a la presentación final del proyecto es obligatoria para todos los alumnos incluidos aquellos que tienen dispensa. Los alumnos con dispensa es recomendable su asistencia a las reuniones de final de Sprint (aproximadamente cada dos semanas). El método de evaluación será el mismo que para el resto de alumnos.

Tabla 4. en esta tabla queda reflejada el tipo de evaluación y el número de actos

\begin{tabular}{|c|l|c|c|}
\hline \multicolumn{1}{|c|}{ TIPO } & \multicolumn{1}{|c|}{ DESCRIPCIÓN } & ACTOS & PESO \\
\hline $\begin{array}{c}\text { (01) Presentación } \\
\text { oral }\end{array}$ & Sprints que se realizan cada dos semanas & 5 & $40 \%$ \\
\hline (11) Observación & $\begin{array}{l}\text { Estrategia basada en la recogida sistemática de datos en } \\
\text { el propio contexto de aprendizaje: ejecución de tareas, } \\
\text { prácticas }\end{array}$ & 1 & $10 \%$ \\
\hline (09) Proyecto & $\begin{array}{l}\text { Supone el análisis y la resolución de una situación } \\
\text { planteada que presenta problemas de solución múltiple, a }\end{array}$ & 1 & $40 \%$ \\
\hline
\end{tabular}


Metodologías ágiles en el grado de Tecnologías interactivas de la Escuela Politècnica Superior de Gandia

\begin{tabular}{|c|l|c|c|}
\hline & $\begin{array}{l}\text { través de la reflexión y el diálogo para un aprendizaje } \\
\text { grupal, integrado y significativo. Se presentará el proyecto } \\
\text { en el último Sprint }\end{array}$ & & \\
\hline $\begin{array}{c}\text { (05) Trabajo } \\
\text { académico }\end{array}$ & $\begin{array}{l}\text { Desarrollo de un trabajo escrito que puede ir desde } \\
\text { trabajos breves y sencillos hasta trabajos amplios y } \\
\text { complejos e incluso proyectos y memorias }\end{array}$ & 1 & $10 \%$ \\
\hline
\end{tabular}

Fuente: EICE, ABP-Diseño y Comunicación, 2018

\subsubsection{Rúbrica para la evaluación del proyecto final}

Para la correcta evaluación del proyecto final se facilita a los alumnos una rúbrica con los conocimientos que debe haber adquirido el alumno, ver tabla 5.

Tabla 5. Rubrica para la evaluación de proyecto

\begin{tabular}{|c|c|c|c|c|c|}
\hline $\begin{array}{l}\text { DEFINIR SUS } \\
\text { OBJETIVOS }\end{array}$ & $\begin{array}{l}\text { Los define y los } \\
\text { identifica } \\
\text { correctamente }\end{array}$ & $\begin{array}{c}\text { Los define, pero no } \\
\text { los identifica } \\
\text { correctamente }\end{array}$ & $\begin{array}{l}\text { Los define pero no } \\
\text { sabe identificarlos }\end{array}$ & $\begin{array}{l}\text { No los define } \\
\text { correctamente }\end{array}$ & $\begin{array}{l}\text { No se han } \\
\text { definido los } \\
\text { objetivos }\end{array}$ \\
\hline $\begin{array}{l}\text { MANEJO DE } \\
\text { INFORMACIÓN }\end{array}$ & $\begin{array}{l}\text { Clasificar y utilizar } \\
\text { la información } \\
\text { correctamente }\end{array}$ & $\begin{array}{c}\text { Clasificar la } \\
\text { información pero la } \\
\text { utiliza } \\
\text { incorrectamente }\end{array}$ & $\begin{array}{c}\text { Clasificar la } \\
\text { información pero no } \\
\text { la utiliza }\end{array}$ & $\begin{array}{c}\text { Clasificar la } \\
\text { información } \\
\text { incorrectamente }\end{array}$ & $\begin{array}{c}\text { No sabe } \\
\text { clasificar la } \\
\text { información }\end{array}$ \\
\hline $\begin{array}{l}\text { INNOVAR Y } \\
\text { CUMPLIR } \\
\text { OBJETIVOS }\end{array}$ & $\begin{array}{l}\text { Responder a los } \\
\text { objetivos e } \\
\text { innovar }\end{array}$ & $\begin{array}{c}\text { Responder a los } \\
\text { objetivos añadiendo } \\
\text { mejoras }\end{array}$ & $\begin{array}{c}\text { Responder a los } \\
\text { objetivos }\end{array}$ & $\begin{array}{l}\text { Dar respuesta a los } \\
\text { objetivos de forma } \\
\text { incorrecta }\end{array}$ & $\begin{array}{l}\text { No dar } \\
\text { respuesta a los } \\
\text { objetivos }\end{array}$ \\
\hline
\end{tabular}

Fuente: EICE, ABP-Diseño y Comunicación, 2018

\subsection{Competencias}

En esta signatura se trabajan específicamente competencias transversales al ser una asignatura que engloba varias asignaturas de distintos departamentos (ver tabla 6).

(cc) EY-NC-ND 2018, Universitat Politècnica de València

Congreso IN-RED (2018) 
Tabla 6. Competencias transversales que se trabajan en la asignatura

\begin{tabular}{|c|c|c|}
\hline MATERIA & COMPETENCIAS TRANSVERSALES & $\begin{array}{l}\text { PUNTO DE } \\
\text { CONTROL }\end{array}$ \\
\hline $\begin{array}{l}\text { Proyecto Diseño y } \\
\text { Programación Web }\end{array}$ & $\begin{array}{l}\text { (08) Comunicación efectiva } \\
\text { - Actividades desarrolladas relacionadas con la } \\
\text { adquisición de la competencia: presentación del } \\
\text { proyecto }\end{array}$ & $\mathrm{Si}$ \\
\hline $\begin{array}{l}\text { Proyecto Diseño y } \\
\text { Programación Web }\end{array}$ & $\begin{array}{l}\text { (12) Planificación y gestión del tiempo } \\
\text { - } \quad \text { Actividades desarrolladas relacionadas con la } \\
\text { adquisición de la competencia: Sprints } \\
\text { - Descripción detallada de las actividades: Periodos de } \\
\text { dos semanas donde el alumno tiene que desarrollar } \\
\text { la solución de los problemas planteados en el sprint } \\
\text { planing para alcanzar el producto mínimo viable } \\
\text { - Criterios de evaluación: Al finalizar el sprint se debe } \\
\text { valorar el resultado obtenido en ese periodo de } \\
\text { tiempo y si ha alcanzado el objetivo planteado en el } \\
\text { sprint planning, cada sprint tiene un valor de un } 8 \%\end{array}$ & $\mathrm{Si}$ \\
\hline
\end{tabular}

Fuente: EICE, ABP-Diseño y Comunicación, 2018

\section{Resultados}

Actualmente los alumnos están trabajando en grupos y desarrollando una dinámica de trabajo muy positiva, como parte de la aplicación de las metodologías ágiles trabajan con tableros de TRELLO (Fig. 1) donde aprenden a organizarse, distribuirse las tareas y administrarse el tiempo, según se establece en el Sprint Planing, lo cual forma parte de la metodología de trabajo de SCRUM, (Nejmeh y Weaver, 2014).

Como podemos ver en la Fig. 1, los alumnos realizan tableros que reflejan el flujo de trabajo, permite comprobar el estado del proyecto y la dinámica de grupo donde la consecución del sprint es lo más importante. Los alumnos saben que cada dos semanas tienen que entregar un producto mínimo viable. Lo que les obliga a mantener reuniones prácticamente diarias para poder trabajar de forma coordinada. Esto les da un alto grado de trabajo en equipo. 
Figura 1: Tablero de Trello de un equipo de trabajo

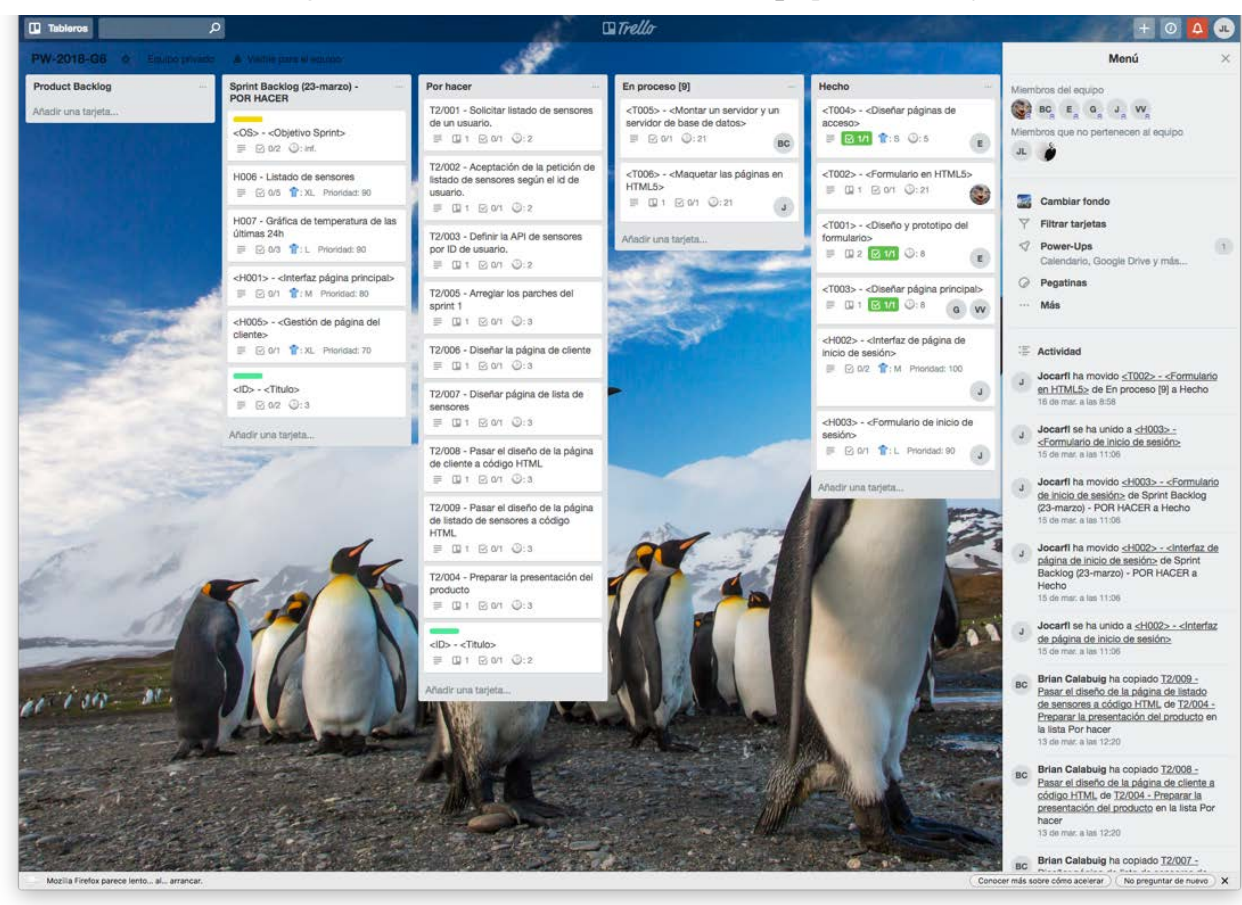

Fuente: EICE, ABP-Diseño y Comunicación, 2018

\section{Conclusiones}

Esta es la primera promoción del grado de Tecnologías interactivas y los resultados que se obtienen son provisionales. Se observa que esta forma de trabajo conlleva que los alumnos organizados en grupos adquieran un alto grado de responsabilidad sobre la ejecución de las tareas, organización y compromiso. Ya que la entrega de un producto mínimo viable cada dos semanas hace que entren en una dinámica de producción continua.

Al ser un proyecto práctico, cuyo resultado es un producto comercializable los alumnos ven como crece. Un producto que han concebido y diseñado ellos mismos, por lo tanto es un equipo motivado. Al mismo tiempo se establecen distintos roles en el equipo ya que debe de ser un equipo multidisciplinar y a lo largo de cada entrega los roles se intercambian entre los miembros del equipo. Por lo tanto están obligados a trabajar en equipo. Ser capaces de asumir el trabajo que ha realizado otro compañero.

Como indicamos anteriormente estas observaciones se realizan mientras los alumnos están trabajando en estos momentos en el proyecto. Deberemos esperar a tener los resultados a finales del semestre para tener datos de la evaluación con personas ajenas al proyecto. Como se indico en la evaluación. Pero los resultados observados invitan a indicar que hay un cambio notable en la actitud de los alumnos a la hora de enfrentarse con el proyecto. 


\section{Agradecimientos}

El desarrollo del presente articulo ha sido realizado gracias al trabajo de los profesores del equipo de innovación y calidad educativa EICE ABP-Diseño y Comunicación de las escuelas, Escuela Técnica Superior de Ingeniería de Diseño, ETSID y la Escuela Politécnica Superior de Gandia, EPSG. También queremos dar las gracias al profesor Jesús Garcia Laborda por sus aportaciones teóricas sobre innovación educativa planteadas.

\section{Referencias}

- AMPUERO CANELlAS, O. CISCAR CUÑA, J. ; DIEZ SOMAVILLA, R.; ENRIQUEZ CARRASCO, E. V.; GONZALEZ DEL RIO COGORNO, J.; .JORDA ALBIÑANA B, MARCH LEUBA, E. (2011) “Aprendizaje basado en proyectos orientado a la comunicación de productos y servicios de diseño”. En IV Jornada de Innovación Docente (JIDINF'11). Valencia, España.

- BAVIERA PUIG A.; CAPO VICEDO J.; GARCÍA FELIX E.V.; GASCH MOLINA E. ; GIMÉNEZ LÓPEZ J.L.; SARASA C.P. (2009). “¿Cómo motivar en el uso de metodologías activas? Algunas experiencias docentes”. En XVII Congreso Universitario de Innovación Educativa en las Enseñanzas Técnicas (CUIEET). (1-9). Valencia, España: ETSI Diseño. Universidad de Valencia

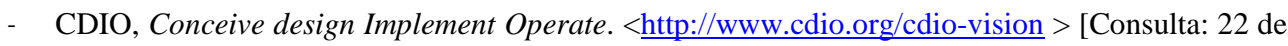
Marzo de 2018]

- FERNANDEZ, E. WILliAMSON, DAVID. M. (2003). "Using Project-based learning to teach object oriented application development “. En CITC4”03. (2003, issue 4, pp. 37-40).

- GUO, R., FARNSWORTH, T. J., \& HERMANSON, P. M. (2016). "Assessment of Evidencebased Management Training Program: Application of a Logic Model.” En International Journal of Management and Business, vol.7, issue1), pp. 57-74).

- HAMMERSLEY, M. (2004), "Some questions about evidence-based practice in education". En G. Thomas \& R. Pring (Eds.). Evidence-based practice in education Maidenhead: Open University Press.

- MANAMENDRA, M.A.S.C., MANATHUNGA K.N., PERERA K.H.D., KODAGODA N.(2013). "Improvements for agile manifesto and make agile applicable for undergraduate research projects”. En 8th International Conference in Computer Science \& Education (ICCSE) 2013 26-28 April 201, Colombo, Sri Lanka. pp. 539-544.

- MAGAL-ROYO T., TORTAJADA-MONTAÑANA I., JORDA-ALMIÑANA B., DEL RIOCOGORNO J. (2006). "El aprendizaje basado en proyectos dentro de la licenciatura de Comunicación Audiovisual”. X Congreso Internacional de Ingenieria de Proyectos . AEIPRO, Valencia 13-15 Septiembre, 2006

- $\quad$ MARKHAM, T., LARMER, J., \& RAVITZ, J. (2003). "Project-Based Learning Handbook: A Guide to Standards Focused Project-Based Learning for Middle and High School Teachers". Novato, CA: Buck Institute for Education.

- NEJMEH B. Y WEAVER S. D. (2014). "Leveraging scrum principles in collaborative interdisciplinary service-learning project courses”. Frontiers in Education Conference (FIE) (2014 IEEE, pp. 1-6).

- PHILIPS R. (1994) "Producing Interactive Multimedia Computer-based learning projects". Computer Graphics (Vol.28, issue1, pp.22-24).

- RICO D. F. Y SAYANI, H. H. (2009) "Use of Agile Methods" En Software Engineering Education. Agile Conference, Chicago pp. 174-179. DOI : 10.1109/AGILE.2009.13

- ROUSSEAU D.M. Y MCCARTHY S. (2007). "Educating Managers From an Evidence-Based Perspective.” Academy of Management Learning \& Education, (Vol. 6, issue 1, pp.84-101)

- ROUSSEAU, D. M. (Ed.). (2012). “The Oxford handbook of evidence-based management”. New York, NY: Oxford University Press 
Metodologías ágiles en el grado de Tecnologías interactivas de la Escuela Politècnica Superior de Gandia

- SALAS, E., WILDMAN, J. L., PICCOLO, R. F. (2009). "Using simulation-based training to enhance management education”. En Academy of Management Learning \& Education, (8, 559573)

- SCRUM. Scrum Organization. < $\quad$ https://www.scrum.org/resources/what-is-scrum $>$ [Consulta: 22 de Marzo de 2018]

- SHERWOOD, A. L. (2004). "Problem-based learning in management education: A framework for designing context”. En Journal of Management Education, (28, 536-557)

- THE AGILE MANIFIESTO, (2018). Agile software development. $<$ http://agilemanifesto.org/iso/es/manifesto.html > [Consulta: 22 de Marzo de 2018]

- TORE D. Y TORGEIR D.(2008). "Empirical studies of agile software development: A systematic review”. En Information and Software Technology. Elsevier. (2008,Vol.50, pp. 833-859). DOI:10.1016/j.infsof.2008.01.006

(cc) EY-NC-ND 2018, Universitat Politècnica de València 